\title{
The value of proximal small intestinal biopsy in the differential diagnosis of chronic diarrhoea
}

\author{
A G Thomas, A D Phillips, J A Walker-Smith
}

\begin{abstract}
The value of proximal intestinal mucosal biopsy was reviewed in 381 children presenting with chronic diarrhoea over an eight year period. An enteropathy was detected in $44 \%$ of cases and was more frequently seen in those aged less than 6 months. A diagnosis was established in $91 \%$ of cases. The most common diagnosis was the postenteritis syndrome where the presence of an enteropathy indicated those requiring treatment with a cows' milk free diet. Other conditions where a biopsy facilitated diagnosis or treatment included giardiasis, enteropathogenic Escheriichia coli, crytosporidiosis, autoimmune enteropathy, and microvillous atrophy.

Coeliac disease was considered in $55 \%$ of children and established in $8 \%$, clearly identifying those requiring a gluten free diet. This also emphasises the important role of the biopsy procedure in the exclusion of specific diseases.

Proximal small intestinal mucosal biopsy is an essential investigation in children with chronic diarrhoea in whom an enteropathy is suspected.
\end{abstract}

Diarrhoea is a major cause of morbidity and mortality in children throughout the world. ${ }^{1}$ The advent of oral rehydration therapy represents a major advance in the management of acute diarrhoea. ${ }^{2-4}$ However, this does not resolve the problem of chronic diarrhoea, which represents a difficult diagnostic problem because of the many causes that can be considered to be responsible for the illness. ${ }^{5}$ The approach at Queen Elizabeth Hospital for Children is to perform a proximal small intestinal mucosal biopsy as a differential diagnostic procedure in children with chronic diarrhoea in whom an enteropathy is suspected. The aim of this paper is to assess the value of such an approach.

Queen Elizabeth Hospital for Children, Hackney Road, London E2 8PS, Academic Department of Paediatric Gastroenterology A G Thomas J A Walker-Smith Electron Microscopy Department A D Phillips Correspondence to: Dr Phillips.
The investigative procedure for these patients included an initial clinical assessment followed by routine microbiological, haematological, and biochemical analyses. Microbiological examination of the stools comprised negative staining electron microscopy for gastrointestinal viruses and light microscopy for ovas, cysts, and parasites. Bacterial culture was performed in order to identify salmonella, Escherichia coli, and shigella. After obtaining informed consent the children were fasted overnight, sedated, and a proximal small intestinal mucosal biopsy specimen obtained using a paediatric twin port biopsy capsule. ${ }^{6}$ Histology, morphometry, ${ }^{7}$ biochemistry, ${ }^{8}$ and ultrastructure ${ }^{9}$ of the small intestinal mucosa were studied depending on the specific clinical requirements. Duodenal juice was examined for the presence of Giardia lamblia. The histological report for each biopsy specimen was reviewed and, for the purposes of this study, graded into normal histology and mild, moderate, or severe enteropathy. ${ }^{10}$ This grading depends mainly on the villous height and crypt depth. Discrete abnormalities such as those associated with lymphangiectasia were included in the normal group as the villous height and crypt depth were normal.

Patients with cows' milk sensitive enteropathy and postenteritis enteropathy were considered together as the diseases may coexist and are difficult to separate without resorting to food provocation and serial small intestinal biopsies. ${ }^{11}$

\section{Results}

During the eight year period 414 children presented with chronic diarrhoea and underwent proximal small intestinal mucosal biopsy. The notes of 381 were retrieved and reviewed retrospectively.

Two hundred and forty two of the patients $(64 \%)$ were aged less than 2 years, $78(20 \%)$ were between 2 and 5 years old, and the remainder $(n=61,16 \%)$ ranged up to 15 years of age. The male to female ratio was $1.4: 1$ (223: 158). Coeliac disease was considered as part of the differential diagnosis in 210 of the children.

Two hundred and twelve children (56\%) had a normal villous height and crypt depth (fig 1); $169(44 \%)$ had an enteropathy, which was mild in $97(25 \%)$ (fig 2), moderate in $38(10 \%)$ (fig 3$)$, and severe in $34(9 \%)$ (fig 4). Enteropathy was more frequent $(\mathrm{p}<0.01)$ in those aged $<6$ months (occurring in 76\% 41/54) and less frequent $(p<0.01)$ in those aged more than 5 years (occurring in 25\%,16/61); analyses by $\chi^{2}$ test.

In the group with normal histology (fig 1) 


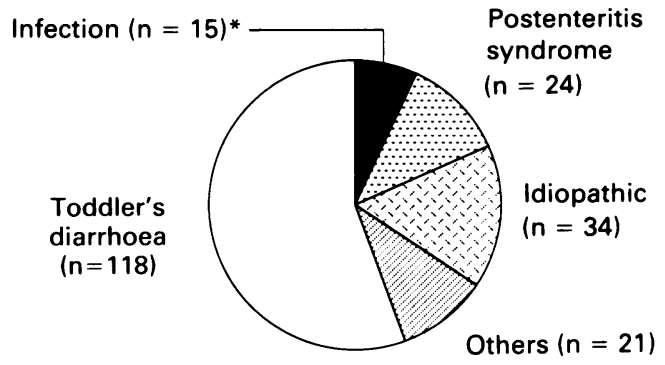

Figure 1 Diagnoses of 212 children with normal histology "For the 15 children with infection the infecting organisms were: salmonella $(n=2), G$ lamblia $(n=5)$, enteropathogenic $E \operatorname{coli}(n=4)$, aeromonas $(n=3)$, and campylobacter $(n=1)$.

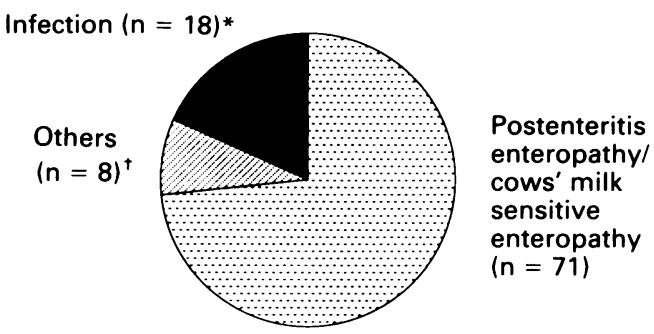

Figure 2 Diagnosesof 97 children withmildenteropathy. "For the 18 children with infection the infecting organisms were: Glamblia $(n=5)$, cryptosporidium $(n=5)$ cryptosporidium $+G$ lamblia $(n=1)$ enteropathogenic $E$ coli $(n=4)$, campylobacter $(n=1)$, and mixed bacteria $(n=2)$. tTheother eight children had inflammatory bowel disease $(n=3)$, coeliac disease $(n=2)$, intractable diarrhoea of infancy $(n=2)$, and idiopathic intestinal pseudo-obstruction $(n=1)$.

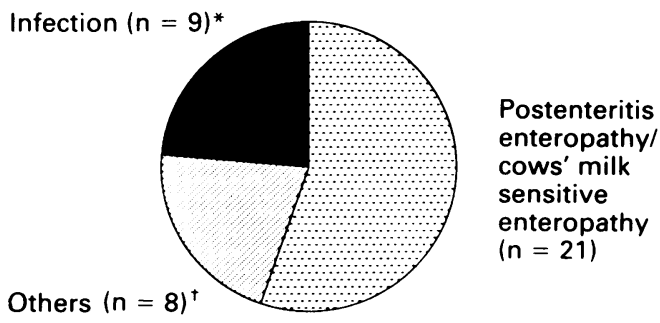

Figure 3 Diagnoses of 38 children with moderate enteropathy. "For the nine children with infection the infecting organisms were: $G$ lamblia $(n=5)$, cryptosporidium $(n=2)$, enteropathogenic $E$ coli $(n=1)$, and mixed bacteria $(n=1)$. + The other eight children had intractable diarrhoea of infancy $(n=2)$, autoimmune enteropathy $(n=2)$, inflammatory bowel disease $(n=1)$, allergic enteropathy $(n=1)$, coeliac disease $(n=1)$, and congenital microvillous atrophy $(n=1)$.

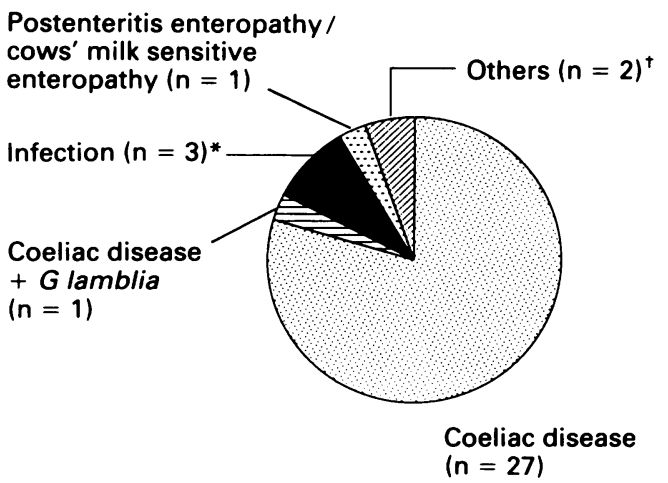
Figure 4 Diagnoses of 34 children with severe enteropathy.
The infecting organism for all three children was $G$ lamblia. tThe other two children had intractable diarrhoea $(n=1)$ and autoimmune enteropathy. toddler's diarrhoea was the commonest diagnosis $(118 / 212,56 \%)$. Twenty four cases $(11 \%)$ were associated with the postenteritis syndrome, that is acute onset of diarrhoea persisting for more than 14 days, with or without failure to thrive. Of these $23(96 \%)$ remained on a normal diet with spontaneous resolution of diarrhoea. In 15 patients $(7 \%)$ bacteria or parasites were demonstrated. Other diagnoses were: food allergy $(n=4)$, cystic fibrosis $(n=3)$, IgA deficiency $(n=3)$, sucrase-isomaltase deficiency $(n=2)$, secondary lactase deficiency $(n=2)$, glucosegalactase malabsorption $(n=1)$, lymphangiectasia $(n=1)$, ileal stricture $(n=1)$, Shwachman's syndrome $(n=1)$, nodular lymphoid hyperplasia $(n=1)$, eosinophilic gastroenteritis $(n=1)$, and inflammatory bowel disease $(n=1)$. No diagnosis was established in 34 children with a normal mucosa (16\% or normal histology group, $9 \%$ of total cases).

Postenteritis enteropathy or cows' milk sensitive enteropathy was the commonest diagnosis associated with mild histological changes ( $n=71,73 \%$; fig 2). In 18 (19\%) cases pathogens, including $G$ lamblia, cryptosporidium, campylobacter, and enteropathogenic $E$ coli, were demonstrated. Other diagnoses included coeliac disease, inflammatory bowel disease, and idiopathic intractable diarrhoea of infancy. The patients with coeliac disease were biopsied on gluten challenge in order to establish the diagnosis after an empirical trial of a gluten free diet at another hospital.

Postenteritis enteropathy or cows' milk sensitive enteropathy was also the commonest diagnosis associated with moderate histological changes $(n=21,55 \%$; fig 3$)$. In nine $(24 \%)$ cases pathogens were demonstrated and in eight (21\%) cases other causes were established.

Severe enteropathy was associated with coeliac disease in $27 / 34$ cases (79\%), giardiasis in three $(9 \%)$, and coeliac disease with giardiasis in one (3\%). Other diagnoses included autoimmune enteropathy, the intractable diarrhoea of infancy syndrome, and the postenteritis syndrome (fig 4).

A total of 20 cases of giardiasis were identified. In 14 cases the parasite was identified by light microscopical examination of duodenal juice and/or small intestinal mucosa and was not seen in stool samples. In three cases giardia was found only in stool samples.

\section{Discussion}

Proximal small intestinal mucosal biopsy affords the demonstration of diffuse, but not discrete, abnormalities, ${ }^{12}$ and in this series $44 \%$ of cases had an enteropathy identified. This alone serves to indicate its importance in paediatric gastroenterology. Its practical value resides not only in its ability to provide differential diagnostic information (when used in conjunction with clinical and laboratory procedures), but also to exclude diagnoses. For example, a diagnosis of toddler's diarrhoea is generally reached on clinical grounds. ${ }^{13}$ However, the patients described here (representing a minority of the total referrals with toddler's diarrhoea during the study period) were suspected of having an 
enteropathy due to coeliac disease or cows' milk protein intolerance. After the demonstration of a normal small intestinal mucosa on a normal diet the diagnoses associated with the presence of an enteropathy were excluded. Similarly although no cause was found to explain the symptoms in $9 \%$ of cases, the biopsy procedure allowed many gastrointestinal diseases to be excluded.

A diagnosis was established in $91 \%$ of cases. The commonest diagnosis was the postenteritis syndrome in $117(31 \%)$. In 24 children a normal mucosa was demonstrated and 23 were continued on a normal diet with resolution of the diarrhoea-that is, the biopsy influenced management with a successful outcome in all but one case. Seventy patients had mild, 21 had moderate, and one had severe histological changes. These children were placed on cows' milk free diets with immediate clinical benefit and several months later a carefully controlled milk challenge was performed in hospital. Most cases were able to tolerate a milk containing diet by the age of 2 years. Thus, the presence or absence of histological changes has helped to indicate which patients with the postenteritis syndrome require treatment with a milk free diet.

In 46 cases pathogens were demonstrated. $G$ lamblia was identified in $20(43 \%)$ and indicated treatment with metronidazole. Proximal small intestinal mucosal biopsy was a more effective method for the detection of $G$ lamblia than stool microscopy. ${ }^{14}$ One patient who did not improve after metronidazole treatment had further biopsies that established the additional diagnosis of coeliac disease. This underlines the value of the procedure in the differential diagnosis of chronic diarrhoea.

Enteropathogenic $E$ coli, when identified adhering to the mucosa, were treated with appropriate antibiotics. ${ }^{15}$ The finding of cryptosporidium explained the presence of an enteropathy ${ }^{16}$ and indicated that therapeutic intervention would be ineffective. In all cases the diarrhoea resolved spontaneously.

Only $8 \%$ of the patients coming to biopsy had coeliac disease, even though it was considered as part of the differential diagnosis in the majority of cases. Thus a major role of the biopsy procedure is to exclude the presence of coeliac disease and thereby avoid the pitfalls of empirical treatment. As treatment of coeliac disease is for life it is crucial that the diagnosis is substantiated.

Other diagnoses associated with a severe enteropathy included autoimmune enteropathy, ${ }^{17}$ congenital microvillous atrophy, ${ }^{18}$ and the idiopathic intractable diarrhoea of infancy syndrome. A small intestinal biopsy is required for the diagnosis of microvillous atrophy and has allowed this discrete disorder to be recognised as part of the differential diagnosis of the intractable diarrhoea of infancy syndrome, ${ }^{18}$ along with autoimmune enteropathy. ${ }^{17}$ Indeed, proximal small intestinal biopsy has facilitated the evaluation of therapeutic trials in these diseases. ${ }^{19-21}$

In conclusion, we have reviewed the use of proximal small intestinal mucosal biopsy in children presenting with chronic diarrhoea. The routine performance of this procedure has established different diagnoses within the umbrella of chronic diarrhoea and influences management. ${ }^{15-18}$ In experienced hands it is a simple and safe procedure and is an essential investigation in chronic diarrhoea.

1 McLaren DS, Cutting WAM. Epidemiology of diarrhoea. Part 1: the global magnitude of diarrhoeal disease in young children. In: Walker-Smith JA, McNeish AS, eds. Diarrhoea and malnutrition in childhood. London: Butterworths, 1986; 203-5.

2 Rahaman MM, Aziz KMS, Patwani Y, Munshi MH. Diarrhoeal mortality in two Bangladeshi villages with and woeal mortality in two Bangladeshi villages with and without communit

3 Anonymous. Oral therapy for acute diarrhoea. Lancet 1981 ;ii: 615-7.

4 Anonymous. Management of acute diarrhoea. Lancet 1983;

$623-5$.
5 Lebenthal E. Chronic diarrhoea in children. Nestlé Nutrition Workshop Series. Vol 6. New York: Raven Press, 1984

6 Kilby A. Paediatric small intestinal biopsy capsule with two ports. Gut 1976;17:158-9.

7 Maluenda C, Phillips AD, Briddon A, Walker-Smith JA. Quantitative analysis of small intestinal mucosa in cow's milk sensitive enteropathy. $\mathcal{F}$ Pediatr Gastroenterol Nutr 1984;3:349-56.

8 Phillips AD, Avigad S, Sacks J, Rice SJ, France NE, WalkerSmith JA. Microvillous surface area in secondary disaccharidase deficiency Gut 1980;21:44-8.

9 Phillips AD, France NE, Walker-Wmith JA. The structure of the enterocyte in relation to its position on the villus: an of the enterocyte in relation to its position on the villus: an
electron microscopical study. Histopathology 1979;3: electron

10 Walker-Smith JA, Phillips AD, Richman PI. Intestinal biopsy. In: Walker WA, Durie PR, Hamilton JR, Walker-
Smith JA, Watkins JB, eds. Pediatric gastrointestinal disease. Smith JA, Watkins JB, eds. Pediatric gastrointestinal
Vol 2. Philadelphia: BC Decker, 1991:1307-23.

11 Spencer J, Isaacson PG, MacDonald TT, Thomas AG, Walker-Smith JA. Gamma/delta T cells and the diagnosis of coeliac disease. Clin Exp Immunol 1991;85:109-13.

12 Lee FD, Toner PG. Biopsy pathology of the small intestine. Biopsy pathology series. London: Chapman and Hall, 1980;32:1421-5.

13 Davidson $M$, Wasserman $R$. The irritable colon of childhood (chronic non-specific diarrhoea syndrome). 7 Pediatr 1966 69: 1027-38.

14 Wright SG, Tomkins AM, Ridley DS. Giardiasis: clinical and therapeutic aspects. $G u t$ 1977; 18:343-50.

15 Hill SM, Phillips AD, Walker-Smith JA. Enteropathogenic Escherichia coli and life threatening chronic diarrhoea. Gut 1991;32:154-8.

16 Thomas AG, Phillips AD, Walker-Simth JA. Cryptosporidiosis in England and Wales. BMF 1990;300:1272-3.

17 Unsworth DJ, Walker-Smith JA. Autoimmunity in diarrhoeal disease. $\mathcal{F}$ Pediatr Gastroenterol Nutr 1985;4:375-80.

18 Phillips AD, Jenkins P, Raafat F, Walker-Smith JA. Congenital microvillous atrophy: specific diagnostic features. Arch Dis Child 1985;60:135-40.

19 Walker-Smith JA, Phillips AD, Walford N, et al. Intravenous epidermal growth factor/urogastrone increases small intestinal cell proliferation in congenital microvillous atrophy. Lancet 1985;ii:1239-40.

20 Drumm B, Cutz E, Tomkins KB, Cook D, Hamilton JR, Sherman P. Urogastrone/epidermal growth factor in treatment of congenital microvillous atrophy. Lancet 1988;i: 111-2.

21 Sanderson IR, Phillips AD, Spencer J, Walker-Smith JA. The response of auoimmune enteropathy to cyclosporin A therapy. Gut 1991;32:1421-5.

\section{Commentary}

This paper comes from a department which has set standards for paediatric gastroenterologists so its conclusions must be treated with respect. Children with diarrhoea lasting more than 14 days, in whom an enteropathy was suspected, underwent small intestinal mucosal biopsy. The authors conclude that this investigative technique is essential in such children.

Apart from providing an interesting insight into the spectrum of chronic diarrhoeal disease as experienced in east London, what message does this paper hold for general practitioners and general paediatricians who, elsewhere in the country, treat the bulk of such patients? 